\title{
Pharmacokinetics of Amoxicillin and Cefepime During Prolonged Intermittent Renal Replacement Therapy: A Case Report
}

\author{
Authors: \\ Jessica H. Xu, ${ }^{1}$ Vesa Cheng, ${ }^{2}{ }^{*}$ Matthew Rawlins, ${ }^{1}$ Joanne Lennon, ${ }^{1}$ \\ David Morgan, ${ }^{3}$ Brett C. McWhinney, ${ }^{4}$ Jacobus P.J. Ungerer, ${ }^{4,5}$ Alice \\ Wilce, ${ }^{4}$ John Dyer, ${ }^{6}$ Jason A. Roberts ${ }^{2,7-9}$ \\ 1. Department of Pharmacy, Fiona Stanley Hospital, Perth, Australia \\ 2. University of Queensland Centre for Clinical Research, Faculty of Medicine, The \\ University of Queensland, Brisbane, Australia \\ 3. Department of Intensive Care Medicine, Fiona Stanley Hospital, Perth, Australia \\ 4. Department of Chemical Pathology, Pathology Queensland, Brisbane, Australia \\ 5. School of Biomedical Sciences, University of Queensland, Brisbane, Australia \\ 6. Department of Infectious Diseases, Fiona Stanley Hospital, Perth, Australia \\ 7. Departments of Pharmacy and Intensive Care Medicine, Royal Brisbane and \\ Women's Hospital, Brisbane, Australia \\ 8. Division of Anaesthesiology, Critical Care, Emergency, and Pain Medicine, Nîmes \\ University Hospital, University of Montpellier, Nîmes, France \\ 9. Centre for Translational Anti-Infective Pharmacodynamics, School of Pharmacy, \\ The University of Queensland, Brisbane, Australia \\ *Correspondence to matthew.rawlins@health.wa.gov.au
}

Disclosure: $\quad$ The authors have declared no conflicts of interest.

Received:

26.03 .20

Accepted:

20.05 .20

Keywords:

Amoxicillin, antibiotics, cefepime, dialysis, dosing, prolonged intermittent renal replacement therapy (PIRRT), sustained low efficiency dialysis.

Citation:

EMJ Nephrol. 2020;8[1]:78-83.

\section{Abstract}

Prolonged intermittent renal replacement therapy (PIRRT) is an emerging form of renal replacement therapy in critically ill patients, but dosing data for antibiotics such as amoxicillin and cefepime are scarce and limited. This case report describes the effect of PIRRT on the plasma pharmacokinetics of amoxicillin and cefepime in a 69-year-old, critically ill patient with a polymicrobial intra-abdominal infection. Blood samples taken over 2 days, including a 7-hour PIRRT session, were analysed and a two-compartment model was used to describe cefepime and amoxicillin clearance and dosing requirements during PIRRT and off-PIRRT in this patient. Based on these data, an off-PIRRT dose of $1 \mathrm{~g}$ amoxicillin 12-hourly and cefepime $2 \mathrm{~g}$ daily with an on-PIRRT dose of $1 \mathrm{~g}$ amoxicillin 8-hourly and cefepime 2 g 12-hourly was deemed appropriate.

\section{INTRODUCTION}

Acute kidney injury and severe infections are common contributing factors to higher mortality

in critically ill patients. ${ }^{1}$ For this patient population, continuous renal replacement therapy (CRRT) has traditionally been used as the form of renal replacement therapy (RRT). However, prolonged 
intermittent renal replacement therapy (PIRRT) is an emerging modality of RRT in the intensive care unit. ${ }^{2}$ PIRRT utilises conventional dialysis machines but runs over a longer time period with lower dialysate and blood flow rates which provides more stable haemodynamics and minimal solute disequilibrium compared to conventional intermittent haemodialysis. ${ }^{1}$ The advantages of PIRRT over CRRT include lower operating costs, decreased workload requirements and risk of infection because of the lack of bag handling, and increased patient mobility and participation in physical and occupational therapy.13,4 CRRT, however, has higher clearance rates of small and large solutes and the use of antimicrobials during CRRT has published references with dosing recommendations. ${ }^{1,4}$ Optimising antimicrobial dosing in critically ill patients is complex due to pathophysiological changes that can alter pharmacokinetics (PK) and pharmacodynamic properties of antimicrobials. ${ }^{1} \quad$ Current antimicrobial PK data during PIRRT are largely limited to case reports or in silico dosing simulation studies. This is further complicated by the variability of PIRRT settings (e.g., blood flow, dialysate, ultrafiltration rates) and haemofilter characteristics, which all contribute to the challenge of antimicrobial therapy optimisation. . $, 5,6^{2}$

The aim of this report is to describe the PK of amoxicillin and cefepime in a patient with polymicrobial intra-abdominal infection undergoing PIRRT and to provide dosing guidance in this setting.

\section{MATERIALS AND METHODS}

\section{Patient Characteristics}

A 69-year-old, $160 \mathrm{~cm}, 90 \mathrm{~kg}$ male presented with perforated sigmoid diverticulitis with faecal peritonitis. He underwent an emergency Hartmann's procedure with formation of an end-colostomy. The patient had a complicated surgical admission; he underwent a further five laparotomies because of ongoing purulent/ faecal collections which culminated into a further large bowel resection and stoma relocation. Initial intraoperative samples grew Escherichia coli, Streptococcus anginosus, Pseudomonas aeruginosa, Enterococcus faecalis, and mixed anaerobic bacteria. A further intraoperative sample taken 10 days later grew E. faecalis and $P$. aeruginosa which was reported to be resistant to piperacillin-tazobactam and meropenem. Wound dehiscence was noted after final surgical closure and swabs of the wound also cultured E. faecalis.

The patient sustained an acute kidney injury upon admission to hospital which temporarily required haemodialysis. Recovery of kidney function occurred 2 months into the patient's hospital admission. During the PK sampling period, the patient was oliguric, with a urine output ranging between $\mathrm{O}$ and $20 \mathrm{~mL} /$ hour.

\section{Antibiotic Dose and Administration}

According to the European Committee on Antimicrobial Susceptibility Testing (EUCAST) interpretive criteria for amoxicillin against E. faecalis and E.coli, clinical isolates are considered susceptible if the minimum inhibitory concentration (MIC) is $\leq 4 \mathrm{mg} / \mathrm{L}$ and $\leq 8 \mathrm{mg} / \mathrm{L}$, respectively, and considered resistant when the $\mathrm{MIC}$ is $>8 \mathrm{mg} / \mathrm{L}$ for both organisms. ${ }^{7}$ Amoxicillin against $S$. anginosus is inferred from benzylpenicillin, for which clinical isolates are considered susceptible if the MIC is $\leq 0.25 \mathrm{mg} / \mathrm{L}$ and resistant if the MIC is $>0.25 \mathrm{mg} / \mathrm{L}^{7}$ Lastly, for cefepime against $P$. aeruginosa and $E$. coli, clinical isolates are considered susceptible if the $\mathrm{MIC}$ is $\leq 0.001 \mathrm{mg} / \mathrm{L}$ and $\leq 1 \mathrm{mg} / \mathrm{L}$, respectively, and resistant when the $\mathrm{MIC}$ is $>8 \mathrm{mg} / \mathrm{L}$ and $>4 \mathrm{mg} / \mathrm{L}_{\text {, respectively. }}{ }^{7}$

The patient was commenced on empirical intravenous (IV) antimicrobials for peritonitis on admission, followed by pathogen-directed therapy once intraoperative microbiology became available. During the PIRRT sampling period, the patient was prescribed IV amoxicillin $2 \mathrm{~g}$ over 30 minutes every 8 hours and IV cefepime $2 \mathrm{~g}$ over 30 minutes every 12 hours. Metronidazole 500 mg given intravenously every 12 hours was also administered in addition to this regimen.

\section{Prolonged Intermittent Renal Replacement Therapy}

PIRRT, using a Fresenius 5008 (Fresenius, Sydney, Australia), was conducted as a 7-hour treatment in the haemodiafiltration mode with a heparinised circuit and a $1.4 \mathrm{~m}^{2}$ filter (Ultraflux ${ }^{\circledR}$ AV6700S, Fresenius), a blood flow rate of $200 \mathrm{~mL} /$ minute, dialysate flow rate of $240 \mathrm{~mL} /$ minute, and an approximate ultrafiltration rate of $233 \mathrm{~mL} /$ hour. 


\section{Blood Sampling}

The patient had a total of eight samples taken over a 2-day period, which included one PIRRT session. Off-PIRRT, blood samples were taken at $0.0,1.0,3.5$, and 6.0 hours post an amoxicillin dose, which also corresponded to a 6.0, 7.0, 9.5, and 12.0-hour (trough) post-cefepime dose. OnPIRRT, blood samples were taken at 0.0, 1.0, 3.0, and 6.0 hours post an amoxicillin dose, which also corresponded to a 3.0, 4.0, 6.0, and 9.0hour post-cefepime dose. Samples were stored at $-80^{\circ} \mathrm{C}$ prior to assay at Pathology Queensland, Brisbane, Australia.

\section{Drug Assay}

Serum amoxicillin and cefepime concentrations were measured by validated high-performance liquid chromatography mass spectrometry at Pathology Queensland. Both analytes were linear from 0.1 to $2.5 \mathrm{mg} / \mathrm{L}$, with an intra- and inter-run precision of $<10 \%$.

\section{Pharmacokinetics Modelling}

PK data were analysed by a nonparametric method with library package for $\mathrm{R}$ and for Pmetrics (Laboratory of Applied Pharmacokinetics, Los Angeles, California, USA) with testing of both one- and two-compartment models. An addition of a second clearance term was included to represent the dialytic clearance from PIRRT, which was tested and included. Both additive $(\lambda)$ and multiplicative ( $\gamma$ ) error models were tested for both drugs. Inspection of the log-likelihood ratio and goodness-of-fit plot was used to select the final models.

\section{Consent}

Informed consent was obtained to collect blood samples and to report this case.

\section{RESULTS}

\section{Pharmacokinetic Results}

The concentrations of amoxicillin during the entire sampling period were at least 5-10fold higher than the breakpoint MIC of $E$. coli ( $8 \mathrm{mg} / \mathrm{L}$ ) and E. faecalis (4 mg/L). ${ }^{7}$ Cefepime concentrations during the sampling were at least 29-fold higher than the breakpoint MIC of $P$. aeuroginosa $(0.001 \mathrm{mg} / \mathrm{L})$ and $E$. Coli $(1 \mathrm{mg} / \mathrm{L})^{7}$ A two-compartment model with clearance terms describing PIRRT clearance and non-PIRRT clearance adequately described the data.

Table 1 describes the mean PK parameters of amoxicillin and cefepime. Figures 1 and 2 provide a graphical representation of the model goodness of fit of amoxicillin and cefepime, respectively, and confirm the adequacy of the model.

Table 1: Mean pharmacokinetic parameters of amoxicillin and cefepime in the patient.

\begin{tabular}{|l|l|l|}
\hline & Amoxicillin & Cefepime \\
\hline $\mathrm{Cl}_{\text {non-PIRRT }}(\mathrm{L} /$ hour) & 8.45 & 5.57 \\
\hline $\mathrm{Cl}_{\mathrm{PIRRT}}(\mathrm{L} /$ hour) & 0.66 & 1.16 \\
\hline $\mathrm{Cl}_{\text {total }}(\mathrm{L} /$ hour) & 9.11 & 6.73 \\
\hline $\mathrm{V}_{\mathrm{C}}(\mathrm{L})$ & 12.58 & 7.10 \\
\hline $\mathrm{K}_{\mathrm{PC}}\left(\right.$ hour $\left.^{-1}\right)$ & 0.45 & 0.52 \\
\hline $\mathrm{K}_{\mathrm{CP}}$ hour $\left.^{-1}\right)$ & 0.47 & 1.01 \\
\hline Error model & $\mathrm{L}=0.2$ (additive) & $\mathrm{L}=0.2$ (additive) \\
\hline
\end{tabular}

$\mathrm{Cl}_{\text {non-PIRRT }}$ : clearance of drug without prolonged intermittent renal replacement therapy; ClPIRRT: clearance of drug with prolonged intermittent renal replacement therapy; $\mathrm{Cl}_{\text {total }}$ : clearance of drug during prolonged intermittent renal replacement therapy with native renal function; $\mathrm{K}_{\mathrm{Cp}}$ : intercompartmental rate constant from central compartment to peripheral compartment; $\mathrm{K}_{\mathrm{pc}}$ : intercompartmental rate constant from peripheral compartment to central compartment; $\mathrm{V}_{\mathrm{c}}$ : volume of distribution of central compartment. 
A)

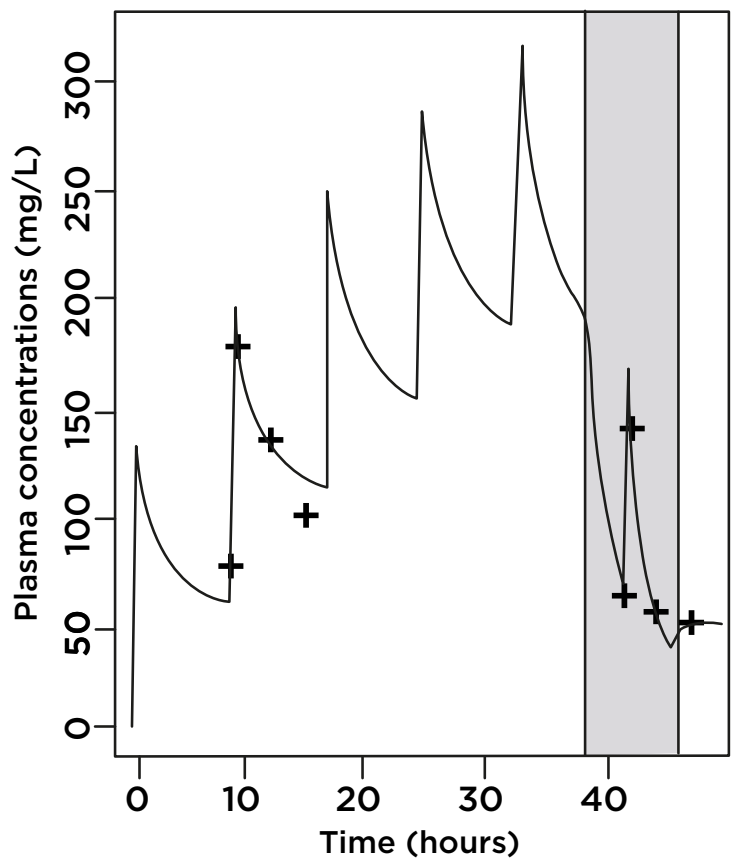

B)

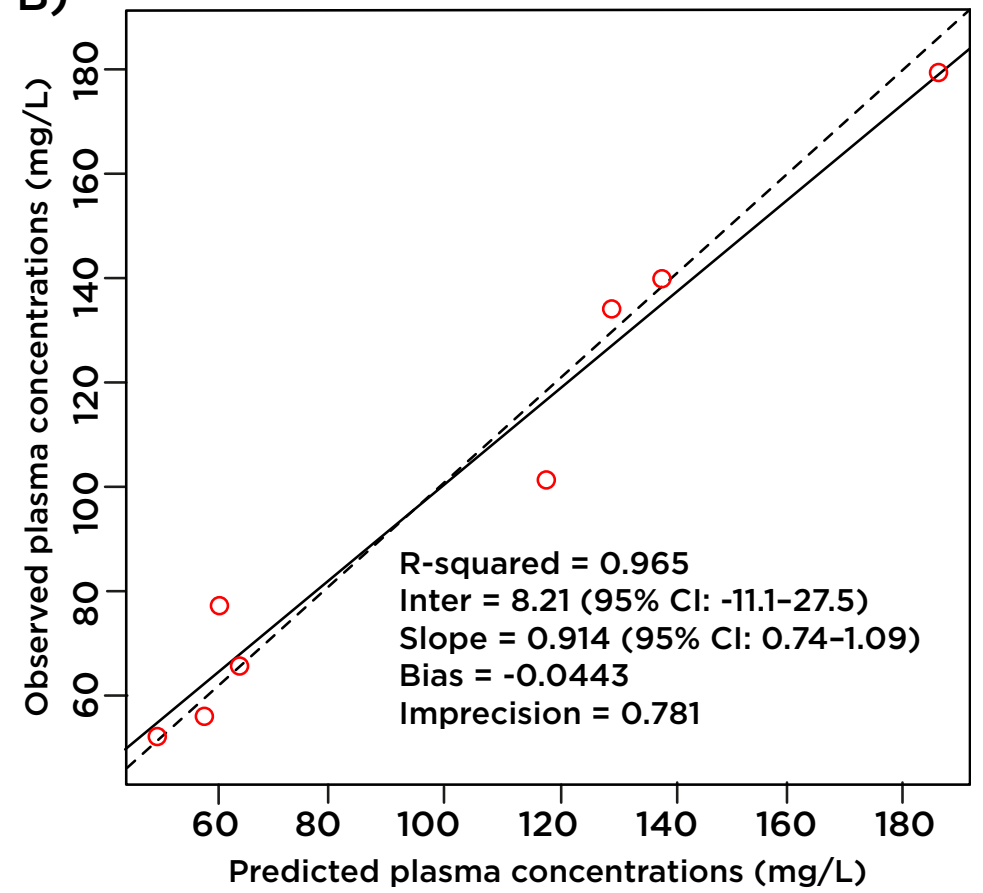

Figure 1: A) The predicted amoxicillin unbound plasma concentration time-profile versus observed data point, with PIRRT therapy shaded in grey. B) Observed-predicted plot for population and individual patient amoxicillin plasma concentrations.

Cl: confidence interval; PIRRT: prolonged intermittent renal replacement therapy.

A)

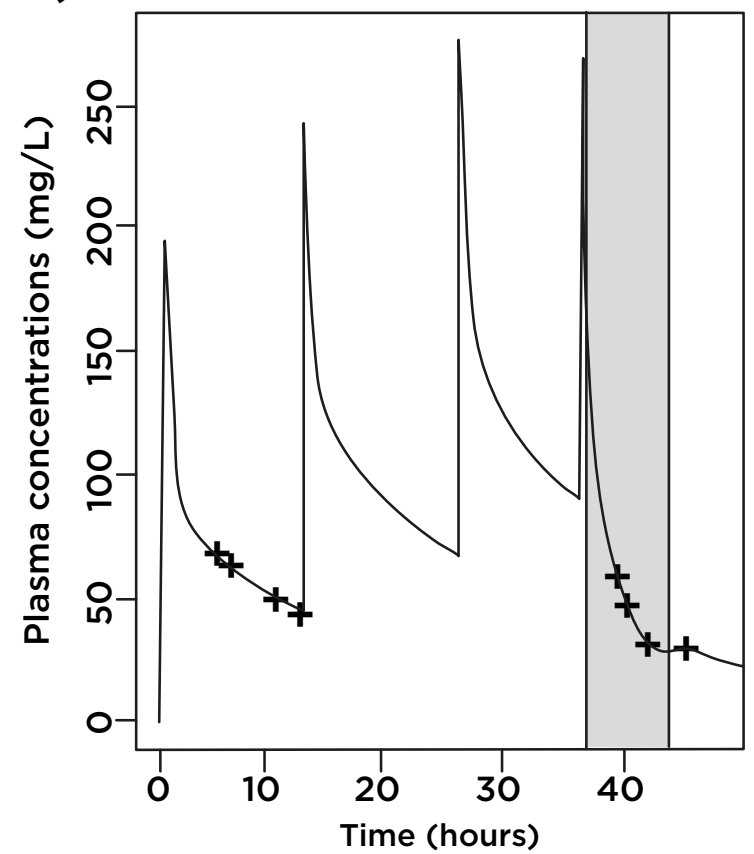

B)

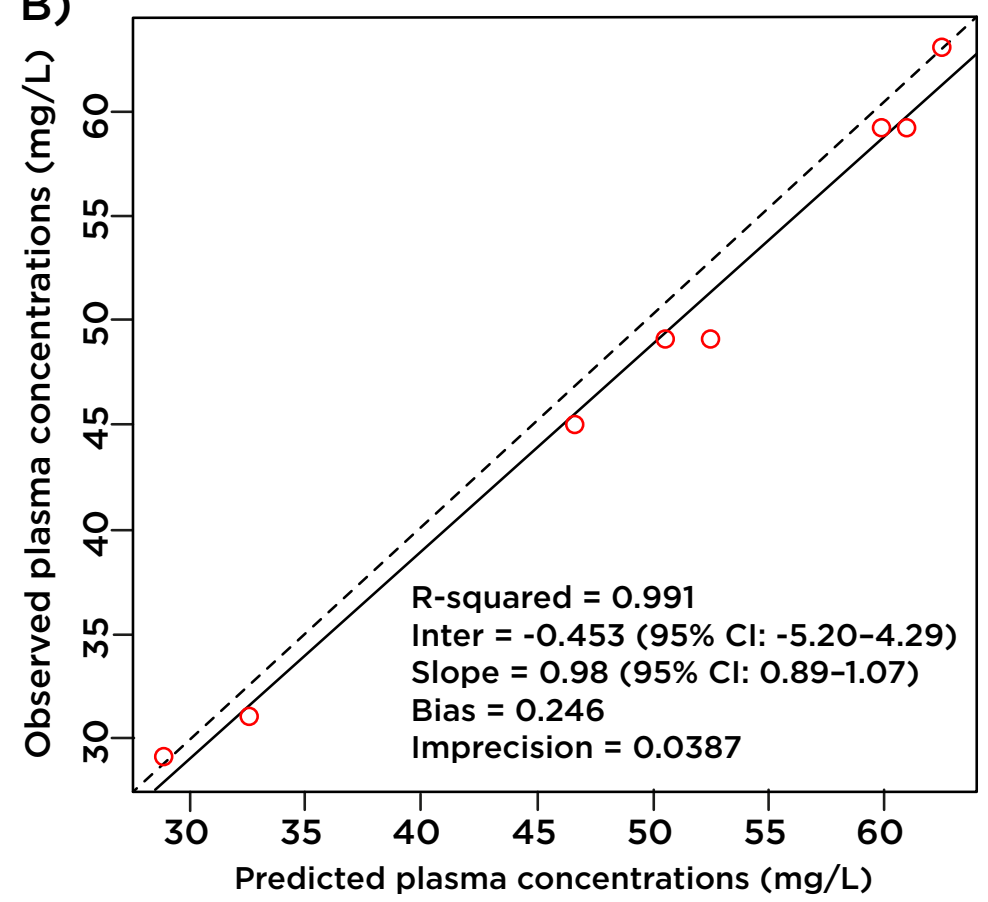

Figure 2: A) The predicted cefepime unbound plasma concentration time-profile versus observed data point, with PIRRT therapy shaded in grey. B) Observed-predicted plot for population and individual patient cefepime plasma concentrations.

Cl: confidence interval; PIRRT: prolonged intermittent renal replacement therapy. 


\section{Clinical Outcomes}

Around the sampling period, the patient exhibited signs of encephalopathy which treating clinicians suspected may have been because of cefepime toxicity. Because of this, ciprofloxacin was substituted for cefepime until antibiotics were subsequently ceased after a total of 6 weeks, with clinical, biochemical, and radiological resolution. The patient was successfully discharged from the intensive care unit after 38 days but remained in hospital to undergo medical treatment, followed by rehabilitation, for a further 6.5 months. He was discharged from hospital to transitional care to await placement in a rehabilitation facility for ongoing care.

\section{DISCUSSION}

To the authors' knowledge, this is the first report describing the effect of PIRRT on amoxicillin PK. Amoxicillin is a $\beta$-lactam antibiotic which is predominantly renally cleared and has been documented to be removed by most forms of RRT. However, dosing recommendations for other RRT modalities cannot be readily transferred to patients undergoing PIRRT. ${ }^{8-13} \quad \beta$-lactams exhibit time-dependent pharmacodynamics, meaning the free drug concentration $(\mathcal{J}$ ) should be maintained over the causative organism's MIC for the maximum amount of time ( $T>M I C)$ to maximise bacterial killing. ${ }^{14}$ Experimental studies recommend a minimum 50\%[T>MIC should be targeted, although higher targets, as high as $100 \%$ JT $>4-5 \times$ MIC, have been suggested in critically ill patients with severe infections. ${ }^{4,15-17}$ In this patient, PIRRT significantly increased amoxicillin clearance compared with periods when PIRRT was not used (9.11 versus $0.66 \mathrm{~L} /$ hour). Because of the high amoxicillin clearance during PIRRT, inadequate amoxicillin dosing while a patient is on PIRRT could potentially lead to antimicrobial resistance or treatment failure. $^{18}$ However, in the sampling period the patient received an amoxicillin dose of $2 \mathrm{~g}$ every 8 hours on both PIRRT and non-PIRRT days, providing sufficient free drug levels to achieve 100\%[T>5xMIC. Given the low amoxicillin clearance when the patient was not on PIRRT and the high plasma levels achieved during the sampling period despite RRT, a dose of $1 \mathrm{~g}$ every 8 hours on PIRRT and $1 \mathrm{~g}$ every 12 hours on nonPIRRT days would be recommended.
Cefepime is a fourth-generation cephalosporin, with antimicrobial activity against Grampositive and Gram-negative bacteria including $P$. aeruginosa, and is known to be removed by RRT. ${ }^{19}$ Like other $\beta$-lactams, cefepime exhibits time-dependent bactericidal activity with studies suggesting similar targets ranging from 60-70\% [T>MIC to $100 \%\left[\mathrm{~T}>4-5 \times \mathrm{MIC}^{19,20}\right.$ There have been various dosing schedules suggested for patients receiving PIRRT, including a Monte Carlo simulation study which recommended a cefepime $2 \mathrm{~g}$ loading dose with $1 \mathrm{~g}$ every 6 hours while on PIRRT or cefepime $2 \mathrm{~g}$ at commencement of PIRRT and $3 \mathrm{~g}$ at the end of PIRRT. ${ }^{21}$ In this patient, PIRRT increased the cefepime clearance significantly (5.57 versus $1.16 \mathrm{~L}$ /hour). In spite of the higher clearance, a therapeutic target of $100 \%[$ T5xMIC was easily achieved during the sample period. The use of cefepime in patients with renal impairment, in the intensive care unit, or of older age has been associated with an increased risk of neurotoxicity which can present as confusion, impaired consciousness, hallucinations, myoclonus, seizures, and encephalopathy. ${ }^{19-24}$ This neurotoxicity is attributed to an ability to cross the blood-brain barrier and exhibit concentration-dependent GABA antagonism. ${ }^{23,24}$ Because this effect is concentration dependent, some studies suggest that a cefepime trough level of $36 \mathrm{mg} / \mathrm{L}$ is a highly accurate and sensitive threshold marker for cefepime-induced neurotoxicity, although this could be as low as $23 \mathrm{mg} / \mathrm{L}^{23,24}$ Supratherapeutic cefepime concentrations could have contributed to the encephalopathy in this case, especially as no dosage adjustments were made when the patient was not being dialysed. A dose reduction to 2 g every 24 hours when not receiving PIRRT to reduce accumulation of cefepime is suggested.

The rate of diffusion, the principle mechanism of drug removal in PIRRT, is proportional to the surface area of the dialyser used. In this study, a dialyser with a surface area of $1.4 \mathrm{~m}^{2}$ was used. Using larger or smaller dialysers would result in proportionally different drug clearances. ${ }^{1}$

\section{CONCLUSION}

In conclusion, amoxicillin $2 \mathrm{~g}$ every 8 hours and cefepime $2 \mathrm{~g}$ every 12 hours both on PIRRT and off PIRRT resulted in concentrations well in excess of the MIC but at levels that could potentially 
be toxic in this patient. The authors recommend giving $1 \mathrm{~g}$ of the amoxicillin dose every 12 hours and $2 \mathrm{~g}$ of the cefepime dose every 24 hours during non-PIRRT periods because of the lower clearance when not on PIRRT. Given individual patient and PIRRT variabilities, however, further data are required to provide dosing recommendations for extrapolation to the rest of the patient population. The impact of PIRRT on metronidazole clearance was not available to be described in this paper.

\section{References}

1. Hoff B et al. Antibiotic dosing for critically ill adult patients receiving intermittent hemodialysis, prolonged intermittent renal replacement therapy, and continuous renal replacement therapy: an update. Ann Pharmacother. 2020;54(1):43-55.

2. Mushatt DM et al. Antibiotic dosing in slow extended daily dialysis. Clin Infect Dis. 2009;49:433-7.

3. Vanholder R et al. Pro/con debate: continuous versus intermittent dialysis for acute kidney injury: a never-ending story yet approaching the finish? Crit Care. 2011;15:204.

4. Sethi SK et al. Antibiotic dosing in sustained low-efficiency dialysis in critically ill patients. Can J Kidney Health Dis. 2018;5:1-12.

5. Jamal $\mathrm{J}$ et al. How can we ensure effective antibiotic dosing in critically ill patients receiving different types of renal replacement therapy? Diag Microbiol Inf Dis. 2015;82(1):92-103.

6. Mei JP et al. Survey of pharmacists' antibiotic dosing recommendations for sustained low-efficiency dialysis. Int J Clin Pharm. 2016;38:127-34.

7. The European Committee on Antimicrobial Susceptibility Testing (EUCAST). Breakpoint tables for interpretation of MICs and zone diameters 2020. 2020. Available at: www.eucast.org. Last accessed: 20 May 2020.

8. Davies BE et al. Pharmacokinetics of amoxycillin and clavulanic acid in haemodialysis patients following intravenous administration of Augmentin. Br J Clin Pharmacol. 1988;26:385-90.
9. Adam D et al. Pharmacokinetics of amoxicillin and clavulanic acid administered alone and in combination. Antimicrob Agents Chemother. 1982;22:353-7.

10. Slaughter RL et al. Effects of hemodialysis on the pharmacokinetics of amoxicillin/ clavulanic acid combination. Ther Drug Monit. 1984;6:424-7.

11. Cheng $\vee$ et al. Pharmacokinetics of benzylpenicillin (penicillin $\mathrm{G}$ ) during prolonged intermittent renal replacement therapy. Chemotherapy. 2019;64:17-21.

12. Fox $E$ et al. Pharmacokinetics of flucloxacillin during prolonged intermittent renal replacement therapy in a 76-year-old man. J Chemother. 2019;31(7-8):419-23.

13. Rawlins $M$ et al. Pharmacokinetics of ceftolozane-tazobactam during prolonged intermittent renal replacement therapy. Chemotherapy. 2018;63(4):203-6.

14. Craig WA. Pharmacokinetic/ pharmacodynamic parameters: rationale for antibacterial dosing of mice and men. Clin Infect Dis. 1998;26:1-10.

15. Garnacho-Montero J et al. Impact of adequate empirical antibiotic therapy on the outcome of patients admitted to the intensive care unit with sepsis. Crit Care Med. 2003;31(12):2742-51.

16. Gonçalves-Pereira J, Póvoa P. Antibiotics in critically ill patients: a systematic review of the pharmacokinetics of beta-lactams. Crit Care. 2011;15:R206.
17. Roberts JA, Lipman J. Antibacterial dosing in intensive care: pharmacokinetics, degree of disease and pharmacodynamics of sepsis. Clin Pharmacokinet. 2006;45:755-73.

18. Keough LA et al. Inadequate antibiotic dosing in patients receiving sustained low efficiency dialysis. Int J Clin Pharm. 2018;40:1250-6.

19. Malone RS et al. Pharmacokinetics of cefepime during continuous renal replacement therapy in critically ill patients. Antimicrob Agents Chemother. 2001;45(11):3148-55.

20. Philpott $C D$ et al. Pharmacokinetics and pharmacodynamics of extendedinfusion cefepime in critically ill patients receiving continuous renal replacement therapy: a prospective, open-label study. Pharmacotherapy. 2019;39:1066-76.

21. Jang SM et al. A Monte Carlo simulation approach for betalactam dosing in critically ill patients receiving prolonged intermittent renal replacement therapy. J Clin Pharmacol. 2018;58(10):1254-65.

22. Chaijamorn $W$ et al. Cefepime dosing regimens in critically ill patients receiving continuous renal replacement therapy: a Monte Carlo simulation study. J Intensive Care. 2018;6:61.

23. Payne LE et al. Cefepime-induced neurotoxicity: a systematic review. Crit Care. 2017;21:276.

24. Lau $C$ et al. A retrospective study to determine the cefepimeinduced neurotoxicity threshold in hospitalized patients. J Antimicrob Chemothe. 2020;75(3):718-25. 